Annales Geophysicae (2002) 20: 741-755 (C) European Geophysical Society 2002

\title{
Evolutions of various solar indices around sunspot maximum and sunspot minimum years
}

\author{
R. P. Kane \\ Instituto Nacional de Pesquisas Espacias, C. P. 515 São José dos Campos, 12201-970, SP, Brazil
}

Received: 2 October 2001 - Revised: 5 February 2002 - Accepted: 12 February 2002

\begin{abstract}
The smoothed monthly sunspot numbers showed that in many solar cycles, (a) during years around sunspot maxima, there was only one prominent maximum, but in some cycles there was a broad plateau. If the beginning and end of these are termed as first and second maxima (separated by several months), the first maximum was generally the higher one, and the valley in between was very shallow. Solar indices at or near the photosphere generally showed similar structures with maxima matching with sunspot maxima within a month or two. Indices originating in the chromosphere and above showed two peaks in roughly the same months as sunspots (with some exceptions, notably the Coronal green line, and the Total Solar Irradiance). Yet often, the second maximum was larger than the first maximum, and the valley between the two maxima was deeper, as compared to sunspot maxima, and (b) during years around sunspot minima, the smoothed sunspot minimum could be sharp and distinct, lasting for a month or two, or could spread over several months. Among the indices originating at or near the photosphere, the Ca K line intensity showed good matching with sunspots, but the Ca Plage area, the Sunspot Group Area, and the solar magnetic fields seemed to show minima earlier than the sunspots, indicating that these activities died out first. These also showed recoveries from the minima later than sunspots. Most of the other indices originating in the chromosphere and corona attained minima coincident with sunspot minima, but in some cases, minima earlier than sunspots were seen, while in some other cases minima occurred after the sunspot minima. Thus, the energy dissipation in the upper part of the solar atmosphere sometimes lagged or led the evolution of sunspots near sunspot minimum. In a few cases, after the minimum, the indices recovered faster than the sunspots. In general, the chromospheric indices seemed to evolve similar to sunspots, but the evolution of coronal indices was not always similar to sunspots, and may differ considerably between themselves.
\end{abstract}

Key words. Solar physics, astrophysics and astronomy

Correspondence to: R. P. Kane (kane@laser.inpe.br)
(Corona and transition region; Magnetic fields; Photosphere and chromosphere)

\section{Introduction}

For the last hundred years or more, the primary index of solar activity has been the Wolf (Zürich) sunspot number $R z$, available since 1700 and published by Wolf in the various issues of Astron. Mitt. (1858-1893) (also in Waldmeir, 1961; McKinnon, 1987) (presently generated by the Solar Index Data Center, Brussels). It has a prominent 11-year cycle, named after Schwabe $(1843,1844)$, who discovered it with less than 11 years of data. The cycles are numbered since 1750 (cycle $1=1755$ minimum to 1766 minimum). In recent years, many other solar indices were established (notably the $2800 \mathrm{MHz}, 10.7 \mathrm{~cm}$ radio emission flux, denoted as F10, recorded routinely by a radio telescope near Ottawa since 14 February 1947, presently operated by the National Research Council using two fully automated radio telescopes at the Dominion Radio Astrophysical Observatory, Penticton, Canada), and all these show an 11-year cycle, except that during sunspot minimum when sunspot numbers almost reach zero, most of the other indices reach a minimum nonzero level.

Solar activity indices show variations in a wide range of time scales, from a few days to several years. In shortterm variations, the most prominent is the 27 -day periodicity, which is attributed to the solar rotation of sunspot groups. Various indices originate in different parts of the solar atmosphere. For example, UV fluxes in the 1600-4000 ̊̊ range originate mostly in the upper photosphere but some in the chromosphere, those from the range 1200-1600 $\AA$ mostly in the chromosphere, $\mathrm{H}$ and $\mathrm{He}$ lines in the upper chromosphere, some Fe lines and X-rays in the corona, and so on. Each region is also a source of radio emissions of certain frequencies, where higher frequencies escape from deeper regions. The magnitudes (percentage changes) of the 27day variation as well as the long-term 11-year variation dif- 
Table 1. List of solar indices used in the present analysis

\begin{tabular}{|c|c|c|c|c|c|c|c|}
\hline Index & Origin & Cyc. 18 & 19 & 20 & 21 & 22 & 23 \\
\hline Years of maximum & & $1946-50$ & $1956-60$ & $1967-71$ & $1978-82$ & 1988-92 & 1997-01 \\
\hline Years of minimum & & $1942-45$ & $1952-55$ & $1963-66$ & $1974-77$ & $1984-87$ & 1994-97 \\
\hline Radio emissions & Corona & & & * & $*$ & $*$ & * \\
\hline Cor. green line & Corona & $*$ & $*$ & $*$ & $*$ & $*$ & * \\
\hline Protons & Corona & & & & & $*$ & * \\
\hline X-rays & Corona & & & * & & $*$ & $*$ \\
\hline F10 & Corona & $*$ & $*$ & $*$ & $*$ & $*$ & $*$ \\
\hline EUV (Pio. Vênus) & Corona & & & & $*$ & $*$ & \\
\hline EUV (Hinteregg.) & Corona & & & & $*$ & & \\
\hline EUV (SOHO) & Corona & & & & & & $*$ \\
\hline UV emissions & Chrom. & & & & & & $*$ \\
\hline PSI $6723 \AA$ & Chrom. & & & & & $*$ & \\
\hline Lyman Alpha & Tra.Reg. & & $*$ & $*$ & $*$ & $*$ & $*$ \\
\hline He I $10830 \AA$ & Chrom. & & & & $*$ & $*$ & $*$ \\
\hline Total Irradiance & Chrom. & & & & $*$ & $*$ & * \\
\hline Mg II $2800 \AA$ & Chrom. & & & & $*$ & $*$ & $*$ \\
\hline Solar Flare Index & Chrom. & & & $*$ & * & $*$ & $*$ \\
\hline Solar Mag. Field & Surface & & & $*$ & $*$ & $*$ & $*$ \\
\hline Sunspot Gr. Area & Surface & & & $*$ & $*$ & $*$ & $*$ \\
\hline Cal. II Plage area & Surface & $*$ & $*$ & * & $*$ & & \\
\hline Cal II K Intensity & Surface & * & $*$ & $*$ & $*$ & $*$ & $*$ \\
\hline Sunspots & Surface & $*$ & * & $*$ & $*$ & $*$ & $*$ \\
\hline
\end{tabular}

fer considerably from one index to another. Donnelly et al. (1985) showed that the solar UV full-disk flux at $2050 \AA$ measured by NIMBUS 7 satellite varied in close agreement with ground-based measurements of the $\mathrm{He} \mathrm{I}$ absorption line at $10830 \AA$ for:

(i) short-term (13.5 or 27.5 days),

(ii) intermediate-term (several months), and

(iii) long-term solar cycle variations, but the UV variations differed from those of the $10.7 \mathrm{~cm}$ solar radio flux F10.

The next major attempt to make a comparative study of the variations of different solar indices was by Donnelly et al. (1986), using the AE-E data of 15 wavelength groups (170-1220 A, Hinteregger et al., 1981), the He I line $10830 \AA$, the F10 flux, and sunspots. Donnelly et al. (1986) showed that the ratio of the amplitudes of the long-term variations to short-term variations was highest (about 2 to 3) for EUV (170-660 $)$ ), about 2 for chromospheric UV, EUV and $10830 \AA \mathrm{He}$ I, and less than 1.5 for F10, $R z$ and Ca-K Plage index. Several details about the 13-day and 27-day variations were presented by them. In her review, Lean (1987) examined all data, namely AE-E data from Hinteregger et al. (1981), the LASP rocket data from
Rottman (1981), Mount et al. (1980) and Mount and Rottman (1981), the SBUV data from Heath and Schlesinger (1984) and the LASP SME data from Rottman and London (1984) and Rottman (1985), and plotted the observational estimates of the ratio of the maximum to minimum UV irradiance during solar cycle 21 . The plots showed a ratio exceeding 2.5 for wavelengths $0-500 \AA$, falling thereafter steadily to $\sim 1.2$ for $1700 \AA$, and below 1.05 for wavelengths exceeding $2100 \AA$. Recently, Kane et al. (2001) subjected the time series of several radio emissions (Crakow Observatory, 275-1755 MHz) to spectral analysis and showed that the coronal region where these radiations originate rotates as a block (same angular velocity) on a 27-day time scale.

A curious feature of the long-term activity is the evolution of sunspot numbers near sunspot maximum. In some cycles, $R z$ rose rapidly to a maximum and fell thereafter rapidly, giving the impression of a sharp peak, while in some other cycles, the rise of $R z$ from the sunspot minimum halted abruptly after a few years, the level remained almost steady for the next few years, giving the impression of a plateau, and then there was a sharp fall up to the next sunspot minimum. Whereas most of the indices had coinciding sharp maxima, in some cases, further evolution was not similar to the sunspot number. For example, in solar cycle 
21 , the smoothed monthly sunspot number reached a maximum value of 165 in December 1979 and dropped thereafter to $\sim 140$ by the end of 1980 . The smoothed F10 had a maximum value of 200.8 in January 1980 (two months after sunspots), dropped to 195.9 in July 1980, but rose thereafter and reached a maximum value of 204.9 in April 1981, 16 months after the sunspot maximum. According to Rybansky et al. (1998), the green Corona Index also showed similar discrepancies and in cycle 21, reached a maximum at the end of 1981. Harvey (1992), Ivanov et al. (1998), Ataç and Ozguç (1998) have pointed out similar dissimilarities.

In the present communication, the behaviours of various indices near sunspot maxima and minima are compared for cycles 18-23.

\section{Data}

In cycle 18 , data for very few indices were available. Thereafter, the number has increased considerably from one cycle to the next. Table 1 lists the indices considered for cycles 18-23, in the order of their approximate altitude of origin in the solar atmosphere, starting from solar radio emissions in solar corona and ending with sunspots at the photosphere.

The short-term variations of solar indices have been studied in detail elsewhere, e.g. in Donnelly et al. (1985, 1986). Here, the emphasis will be on what Donnelly et al. (1985) termed as intermediate-term time scale. Hence, only monthly means are used and further, 12-month moving averages are calculated and used. Most of these data were available in the NOAA website ftp://ftp.ngdc.noaa.gov/STP/SOLAR DATA/, (henceforth referred as NGDC) but some were from other websites mentioned by Helen Coffey and/or by the respective authors, and some were obtained privately. Many are in the NOAA Publication Solar Geophysical Data (SGD) Comprehensive Reports.

\section{Plots of sunspot numbers for cycles 0-23}

Figure 1 shows a plot of the smoothed sunspot number $R z$ for all the cycles 0-22 (cycle 23 partially). Two categories are seen:

(a) cycles with one prominent maximum: cycles $0,1,2,3$, $4 ; 7,8,9,10,11,12,13 ; 15 ; 18,19$;

(b) cycles having a broad plateau (the beginning and the end could be considered as two maxima): cycles 5, 6; 14; 16,$17 ; 20,21,22$. In this category, the first maximum is generally larger than the second maximum, and the valley in between is very shallow.

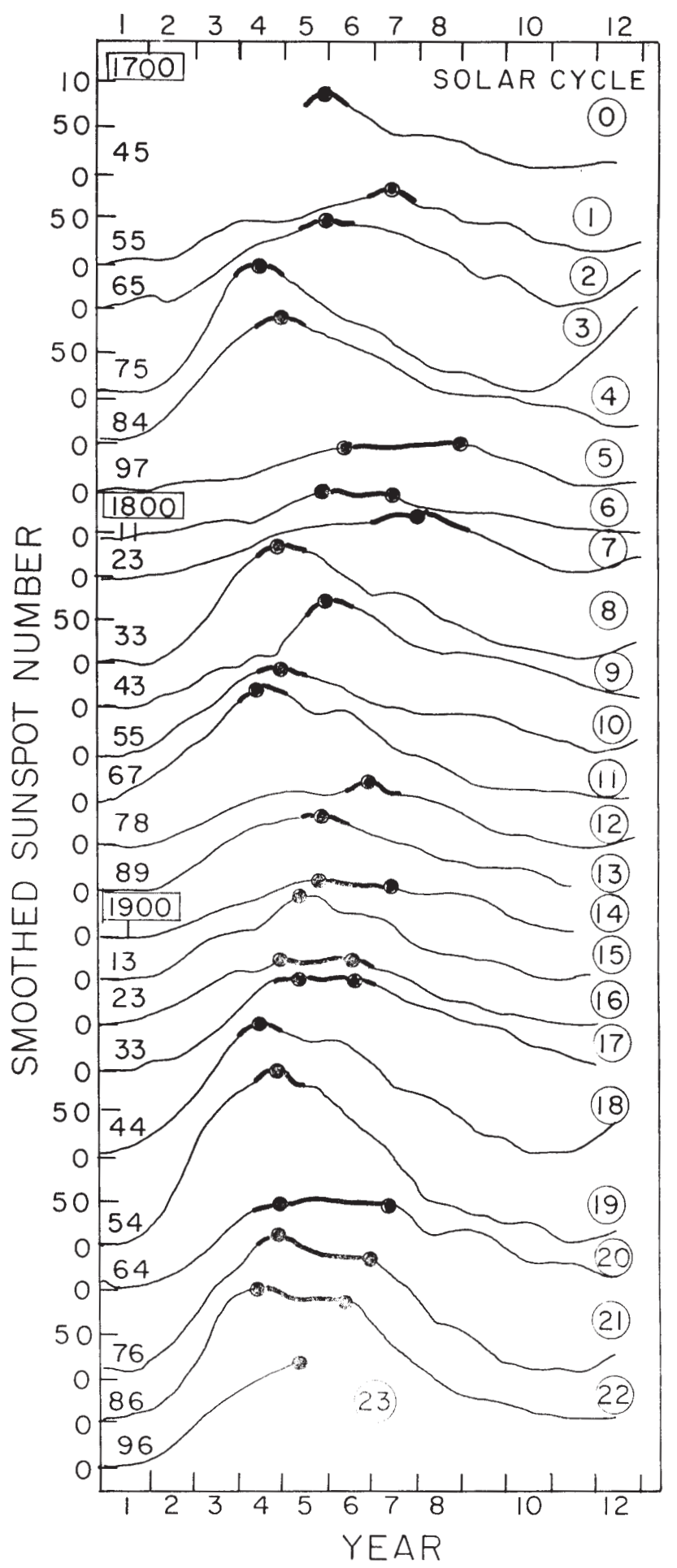

Fig. 1. Plot of smoothed monthly sunspot numbers for cycles $0-23$. The full dots indicate maxima.

\section{Years around sunspot maxima}

\subsection{Plots for cycles 18-23}

Figure 2 shows plots for (a) Cycle 18 and (b) Cycle 19. The indices for which data were available are: 

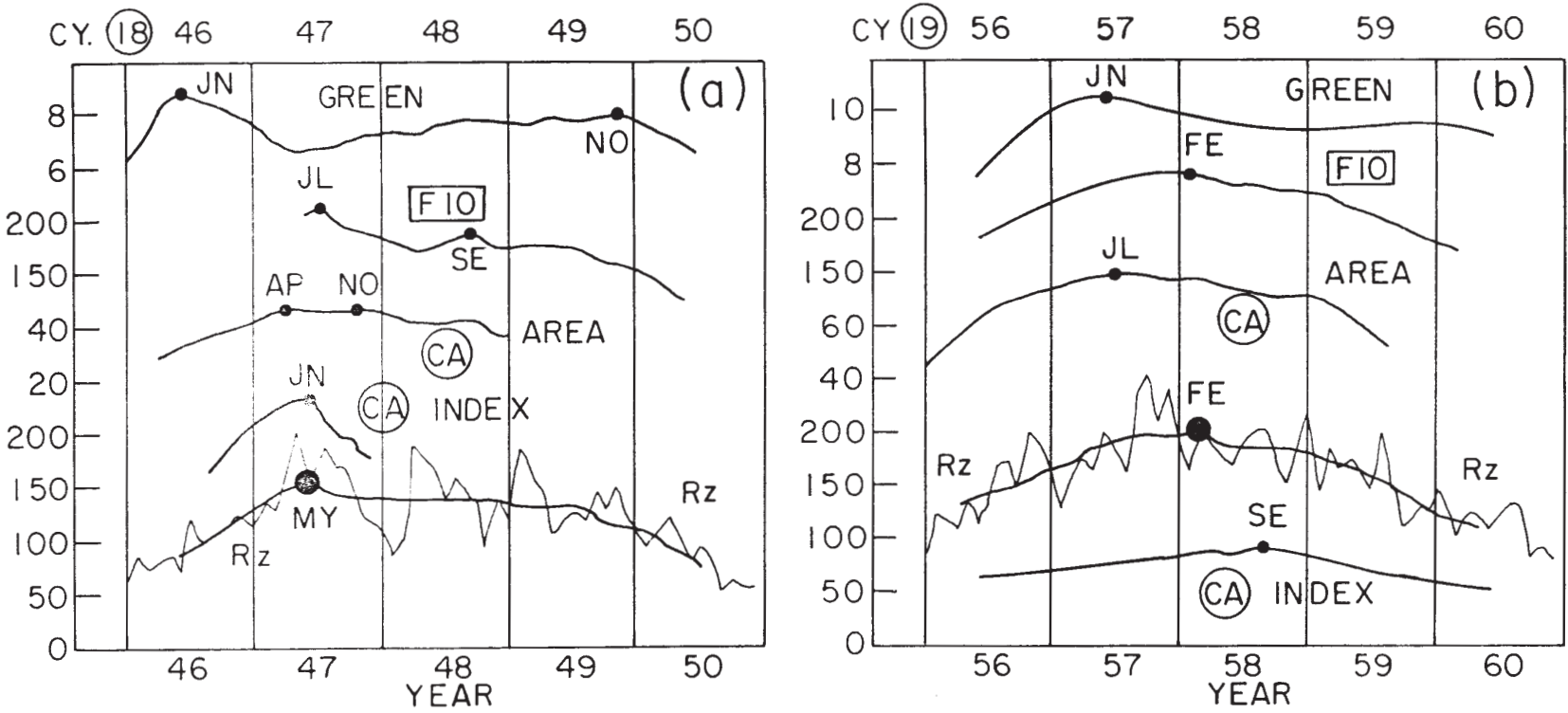

Fig. 2. Plots of various solar indices in years near sunspot maxima of (a) cycle 18, 1946-1950, and (b) cycle 19, 1956-1960. The full dots indicate maxima.

(i) the sunspots, NGDC,

(ii) Mt. Wilson Observatory Calcium Plage Area, Foukal (1996), NGDC,

(iii) the Calcium K line intensity, McMath, Sacremento Peak, Big Bear Observatories, Swartz and Overbeck (1971), NGDC,

(iv) the $10.7 \mathrm{~cm}(2800 \mathrm{MHz})$ flux denoted as F10, NGDC, and

(v) the Coronal green line (Fe XIV, $5303 \AA$ ) index (Rybansky et al., 1998; Ramesh, 1998), NGDC.

In cycle 18, the smoothed sunspots $R z$ had a maximum in May 1947. The Ca Plage area had a broad maximum around the same period, but the $\mathrm{Ca}$ index had a sharp maximum in June 1947. The F10 had a main maximum in July 1947 and a subsidiary maximum in September 1948. (The maxima are indicated by full dots and months of the various maxima are indicated by two letters as JA, FE, MA, AP, MY, JN, JL, AU, $\mathrm{SE}, \mathrm{OC}, \mathrm{NO}, \mathrm{DE}$ ). The Coronal green line index (smoothed values) had a prominent maximum in June 1946, almost a year before the sunspot maximum. In addition, the green line had a secondary maximum much later, in November 1949. Thus, in cycle 18, the Coronal green line index had a development very different from the other indices. In cycle 19 (Fig. 2b), the sunspot maximum occurred in February 1958, the F10 maximum coincided with the same, the Ca Plage area and Coronal green line maxima occurred 6-8 months earlier, and the $\mathrm{Ca} \mathrm{K}$ line intensity index maximum occurred $\sim 7$ months later, but all indices had only one prominent maximum. Interestingly, the $\mathrm{Ca}$ intensity index had a very different behaviour in these two cycles. In both, the sunspots had a broad peak, but the $\mathrm{Ca}$ index peak was sharp in cycle 18 and broad in cycle 19 . Thus, parallelism between the Ca intensity index and the sunspots is not invariably there. In contrast, the Ca Plage area was broad in both the cycles.

Figure 3 shows a plot of the smoothed values of sunspot number $R z$ and the Ca Plage area for the previous 3 cycles $15,16,17$. As can be seen, with slight differences, the two indices run parallel.

Figure 4 shows the plots for cycle 20. Here, additional indices are:

(i) solar magnetic field represented by the Magnetic Plage Strength Index (MPSI) derived from spatially resolved magnetogram observations at the 150-foot solar tower at Mt. Wilson Observatory (MWO) (Parker et al., 1998), full line, website http://www.astro.ucla.edu/ obs/intro. html,

(ii) monthly counts of grouped solar flares (SGD),

(iii) hard and soft X-rays (SOLORAD, GOES satellites), NGDC, and

(iv) radio emissions at 609, 1415, 2695, 4995, 8800, 15400 MHz (SGMR, Sagamore Hill; PALE, Palehua, Hawaii; LEAR, Learmonth, Australia; SVTO, San Vito, Italy), NGDC.

The sunspots had a major maximum in October 1968, and a minor maximum in June 1970. The Ca Plage area and Ca index also had two maxima each, but several months earlier than the sunspot maxima. There is another index, namely the corrected Sunspot Group Area, measured and distributed by the solar group of the Rome Astronomical Observatory (data available in NGDC). In Fig. 4, the full triangles near the $\mathrm{Ca}$ Plage Area index plot represent the maxima. The first maximum was in April 1968, later than that of the Ca Plage area 


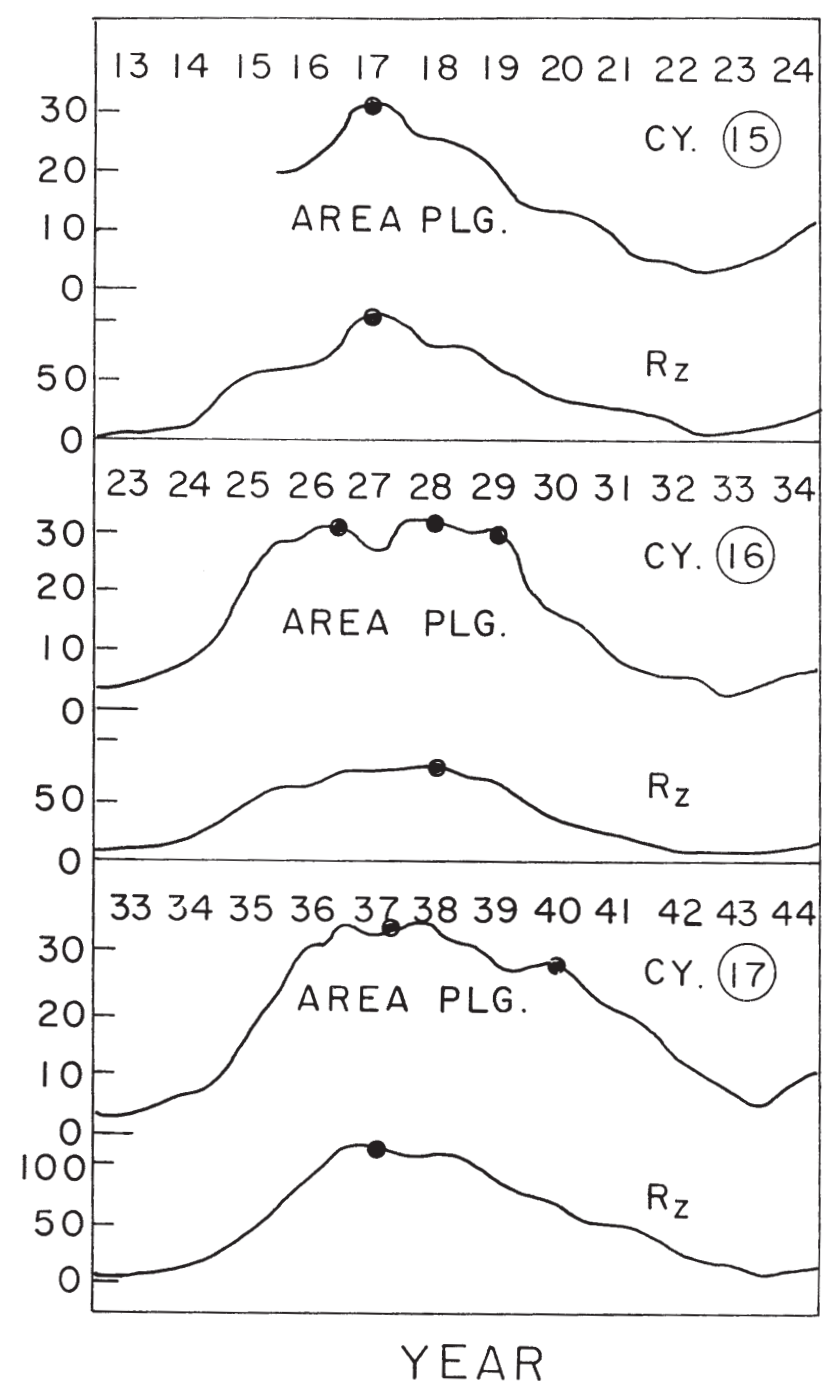

Fig. 3. Plots of smoothed sunspot number $R z$ and the Ca Plage Area, for cycles $15,16,17$. The full dots indicate maxima.

index (May 1967), almost the same as that of the Ca intensity index (March 1968), but much earlier than the sunspot maximum (October 1968). The second maximum was in May 1970 and almost coincided with the small second maximum of sunspots (June 1970). Thus, for cycle 20, the Ca Plage area had a behaviour very different from those of earlier cycles. The solar magnetic field index MPSI started late, in the middle of 1970 and only one maximum could be seen, in July 1970. The grouped solar flare count had two maxima, in August 1967 (several months before the first sunspot maximum of October 1968), and in July 1970 (almost coincident with the second sunspot maximum in June 1970). The soft (1-8 $\AA$ ) and hard (8-20 ̊) SOLORAD X-ray data showed a maximum in June 1970. The F10 had two maxima, in January 1968 and July 1970. The Coronal green line had two maxima, in June 1968 and June 1970, but only the second maximum coincided with the second maximum of sunspots (June 1970), while the first maximum occurred sev-

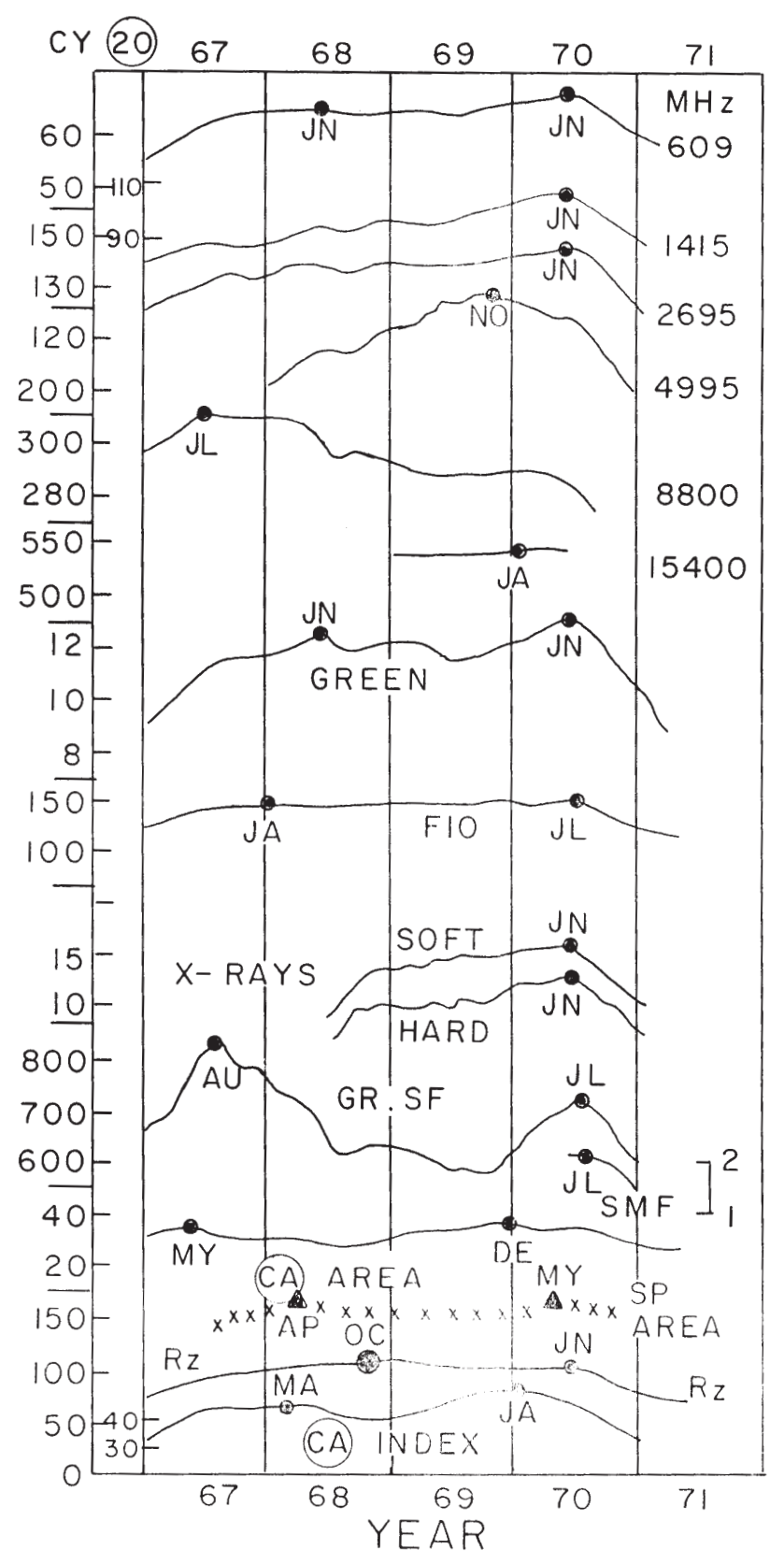

Fig. 4. Plots of smoothed values of the various solar indices in years near sunspot maximum of cycle 20, 1967-1971. The full dots indicate maxima.

eral months earlier than the first sunspot maximum (October 1968). In the radio emissions, $609 \mathrm{MHz}$ had a major maximum in June 1970 and a minor maximum in June 1968, while 1415 and $2695 \mathrm{MHz}$ had just one maximum in June 1970, $4995 \mathrm{MHz}$ in November $1969,8800 \mathrm{MHz}$ in July 1967, and $15400 \mathrm{MHz}$ in January 1970. Interestingly, none of these indices had any maximum at the October 1968 maximum of the sunspot number, but among themselves, some had a maximum in 1967, and many had a maximum at or near the second (minor) sunspot maximum in June 1970. Thus, whereas the sunspot may show a major maximum followed by a mi- 
nor maximum, some indices seem to show a prominent maximum at or near the second rather than the first sunspot maximum.

Figure 5 shows the plots for (A) cycle 21 and (B) cycle 22 . In cycle 21 (Fig. 5a), there are many additional indices. In particular, a whole set of $15 \mathrm{UV}$ and EUV emissions were available (Hinteregger et al., 1981; Donnelly et al., 1986, NGDC). Some of these had wavelengths very near each other. The percentage deviations of these were plotted and those showing similar variations were combined together. Finally, the wavelengths or ranges chosen were: Lyman Alpha (1216 ̊), Lyman Beta (1026 ̊), 510-660 ̊, 206- $304 \AA$, and 168-204 $\AA$. Besides these, there were the composite values of:

(i) Mg II (2800 ̊̊, Deland and Cebula, 1993), NGDC,

(ii) Total Solar Irradiance (Frohlich and Lean, 1998), data from PMOD/WRC and VIRGO team, NGDC,

(iii) He I absorption line (10830 ̊, website ftp://argo.tuc. noao.edu/kpvt/daily/stats/he.dat), and

(iv) Pioneer Venus Orbiter integrated EUV flux (300$1220 \AA ̊$, Brace et al., 1988; Mahajan et al., 1998, NGDC, updated data sent privately by K. K. Mahajan).

Lyman Alpha data were also available as a modelled series since 1947 through 1999 (Woods et al., 2000), but their estimations are partly based on other indices (Mg II, etc.). In the radio emissions, additional data were available for 245 and $410 \mathrm{MHz}$., NGDC. In cycle 22 (Fig. 5b), additional data were available for

(i) Solar protons (GOES, NGDC),

(ii) X-rays (GOES, NGDC), and

(iii) The Photometric Sunspot Index (PSI, line 6723 Chapman et al., 1994, NGDC).

For cycle 21 (Fig. 5a), the sunspots had a major maximum in December 1979 and a minor maximum in August 1981. The Ca index showed only one peak in January 1980. The Ca Plage area had two peaks, one in July 1979 (earlier than the December 1979 peak of sunspots) and another in September 1981, very near the August 1981 peak of sunspots. The Sunspot Group Area maxima (full triangles) were in November 1979 and September 1981, very near the sunspot maxima. For the solar magnetic field, besides the Mt. Wilson observations (full line), Kitt Peak line of sight magnetic field strength data (dashed line) were available, (website $\mathrm{ftp} / / /$ argo.tuc.noaa.edu/kpvt/daily/stats/mag.dat). Both these showed a peak in July 1979, similar to the Ca Plage area (earlier than the sunspot peak), but the second peak for Mt. Wilson was in September 1981, while that of Kitt Peak was in April 1981. (K. Harvey sent us another set of Kitt Peak magnetic data integrated over the Sun by Carrington rotations. The 13-value moving averages of the Harvey data set gave the same maxima, within a month, as the other Kitt
Peak set). This difference may be insignificant but could also reflect different responses of the two magnetic indices (Mt. Wilson and Kitt Peak) to solar energy convection. The grouped solar flare index had two peaks a few months earlier than the sunspot peaks. For solar flare index, additional data were available from the Kandilli Observatory, Istanbul, Turkey (Ataç and Ozguc, 1998), NGDC), separately for the Northern (NH) and Southern (SH) Hemispheres of the Sun. The data were available only from 1980 onwards, but the two hemispheres show very different results, with the NH having just one peak in January 1981, and SH having two peaks, in May 1980 and December 1982, none of these tallying with any of the sunspot peaks. The Mg II index and He 10830 had two peaks, the first one coinciding with the first peak of sunspot and the second peak a few months away from the second peak of sunspot. The Total Solar Irradiance (crosses) showed a small peak in July 1979, and a major peak in December 1980, both of these were several months earlier than the sunspot peaks. The modeled Lyman Alpha had a minor peak in January 1980 and a major peak in July 1981, both matching with sunspot peaks. The Hinteregger data were up to the middle of 1980 only. Hence, the moving averages of Lyman Beta and the EUV revealed only one peak in December 1979-January 1980, matching well with the sunspot peak. However, the Lyman Alpha of Hinteregger showed a peak in June 1979, several months earlier than the sunspot peak. The Pioneer-Venus EUV showed just one peak, which was a few months earlier than the second sunspot peak. The F10 had two peaks matching well with sunspots. The Coronal green line had only one major peak a few months later than the second sunspot peak. Most of the radio emissions had two peaks matching well with the sunspot peaks, but $15400 \mathrm{MHz}$ had a peak in April 1979, several months earlier than the sunspot peak. By and large, many indices showed two peaks matching reasonably well with the sunspot peaks. But, whereas the second peak was minor for sunspots, it was a major peak for some indices. Also, some indices showed first peaks earlier than the first sunspot peak $(15400 \mathrm{MHz}$, Total Solar Irradiance, Solar magnetic field, Ca Plage area), while some indices showed second peaks earlier than the second sunspot peak (Total Solar irradiance, Grouped solar flare count).

For cycle 22 (Fig. 5b), the sunspots had a major peak in July 1989, and a diffuse peak during January-August, 1991. The Ca II intensity index (PFIFX of K line Facular data, Chapman et al., 1996, NGDC) showed peaks similar to those of sunspots. No data were available for the $\mathrm{Ca}$ Plage area, but the sunspot group area (full triangles) showed maxima in May 1989 and February 1991, almost matching with the sunspot maxima. Many other indices had two peaks. The Coronal green line had only one peak coinciding with the first major peak of sunspots, but Lyman Alpha, He I $10830 \AA$, and the Total Solar Irradiance had the first peak 5-6 months later than the first sunspot peak of July 1989. The solar magnetic field at Mt. Wilson (full line) had one peak in March 1990 and another in August 1991, but the magnetic field at Kitt Peak (dashed line) showed one peak 


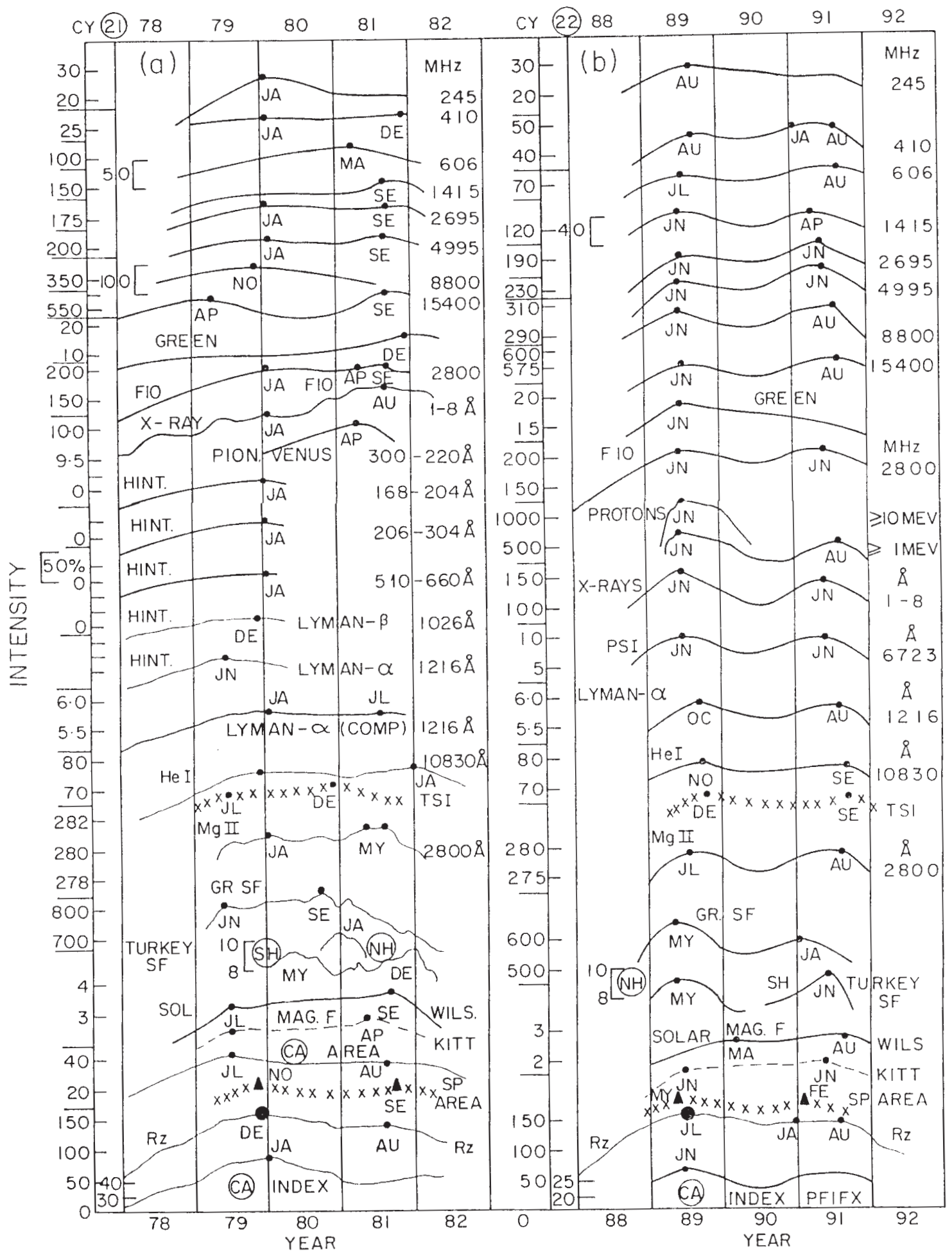

Fig. 5. Plots of smoothed values of the various solar indices in years near sunspot maxima of (a) cycle 21, 1978-1982, and (b) cycle 22, 1988-1992. The full dots indicate maxima.

in June 1989 and another in June 1991, both matching well with sunspots. Again, there seems to be some difference in the responses of the two magnetic field indices (Mt.Wilson and Kitt Peak). The grouped solar flares had peaks matching with sunspots, but the Turkey solar flare indices showed very different behaviours for the $\mathrm{NH}$ and $\mathrm{SH}$ solar hemispheres, the NH having just one peak in May 1989 (almost the same as the sunspot peak of July 1989), while the SH had just one 
peak in June 1991 (almost the same as the sunspot peak near August 1991). The difference in the indices of $\mathrm{NH}$ and $\mathrm{SH}$ could be a major factor in the evolution of sunspot activity near the solar maximum. By and large, the matching between the various indices and sunspots was better in cycle 22 , than in earlier cycles.

Figure 6 shows the plots for cycle 23. These are incomplete as data for many indices were not yet available. ( $\mathrm{SOHO}$ data, $\sim 300 \AA$ were on the website http://www.usc.edu/dept/ space_science/semdata.htm, courtesy of D. McMullin). On the other hand, a whole set of SOLSTICE chromospheric line data were available (courtesy of T. Woods of LASP, Boulder, Colorado). The sunspots (smoothed values) showed a maximum in March 2000, and many other indices for which data were available up to 2001 also showed a maximum thereabout or slightly later. How would the cycle 23 evolve in the future is anybody's guess. Like cycles 3, 11, 18 (see Fig. 1), a decline may follow. Or, like some other cycles, the present level may be maintained approximately for the next 2-3 years (a plateau), and a decline may follow thereafter. In that case, a second (minor?) sunspot maximum may occur and some of the other indices may show similar features (two maxima) but in different relative proportions.

\subsection{Long-term variation of the maxima of some solar in- dices}

Rybansky et al. (1998) mention that the Coronal green line index in the maxima of different solar cycles shows a monotonic increase since 1939. There are very few indices for which continuous data are available for many cycles. Figure 7 shows some plots of the maxima of smoothed values. The sunspots increased from cycle 15 to cycle 19 and have a decreasing tendency thereafter. The Calcium Plage Area (also the Sunspot Group Area) and the $2800 \mathrm{MHz}$ F10 show similar tendencies. The $\mathrm{Ca} \mathrm{K}$ line intensity index seems to have a downward tendency since cycle 18, and the Coronal green line index rose monotonically since cycle 18 .

The Coronal green line index patterns often seem to deviate considerably from the sunspot number patterns (Figs. 2 and 7). The reason for this peculiar behaviour needs further exploration. Ramesh (1998) made correlative studies of the Coronal green line index with sunspots and reported good correlation on a yearly average basis, which we did not observe invariably. Rybansky et al. (1998) did not find any significant correlation between the Coronal green line index and solar soft X-rays and attributed this to the difference in the size of the coronal regions in which these indices originate (small-scale regions for X-rays, larger regions for green line emission). Incidentally, the Total Solar Irradiance also seems to show considerable dissimilarities with sunspots, as shown here and also in Ataç and Ozguc (1998).

\subsection{Conclusions about years around sunspot maxima}

From the analysis presented so far, the following conclusions can be drawn:

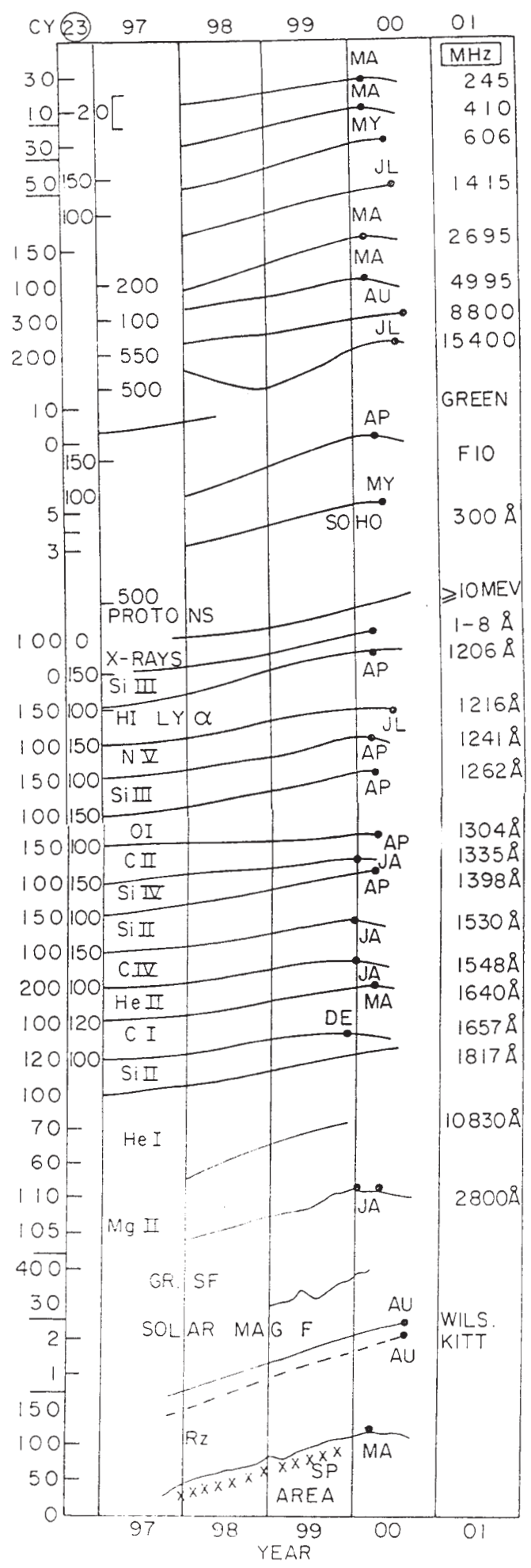

Fig. 6. Plots of smoothed values of the various solar indices in years near sunspot maximum of the present cycle 23, 1997 onwards. The full dots indicate maxima.

(1) The monthly values of sunspot numbers show large, mostly erratic month-to-month variations. But their 12month moving averages show distinct patterns near the sunspot maxima. 

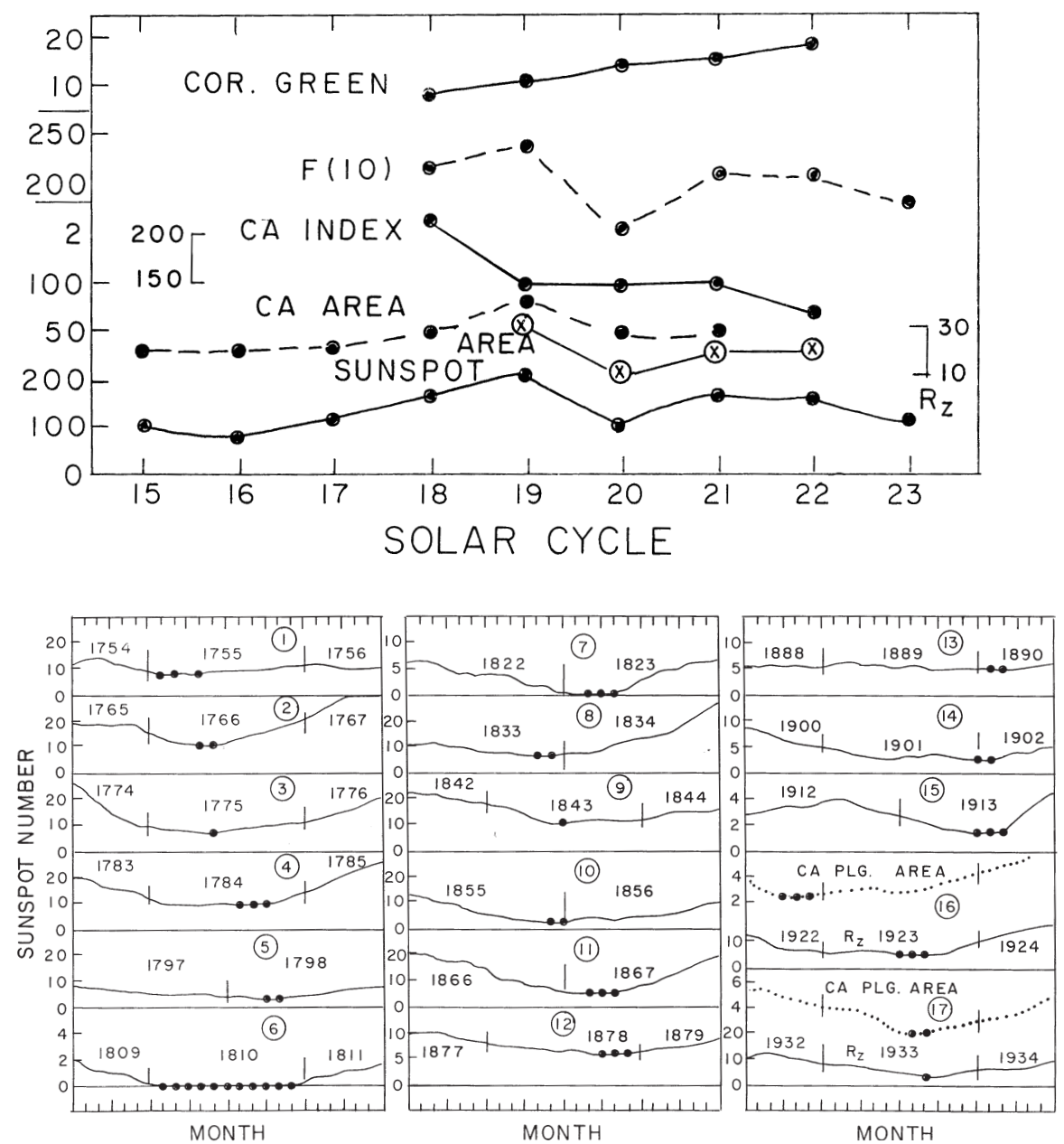

Fig. 7. Plots of smoothed values of Coronal green line index, F10, Calcium $\mathrm{K}$ line intensity index, Calcium Plage Area and Sunspot Group Area, and sunspot number, at sunspot maxima of cycles 15-23.
(2) In many (11-year) cycles, there is only one prominent sunspot maximum, but in some cycles, there is a broad plateau. If the ends are termed as first and second maxima, the first maximum is invariably larger. The valley between the two maxima is very shallow. Indices originating at or near the photosphere show similar patterns.

(3) When there are two maxima in sunspots, most of the other indices also show two maxima within a month or two of the sunspot maxima. However, in contrast to sunspots where the second maximum is equal or smaller, the second maximum in the other solar indices is sometimes bigger than the first maximum.

Also, the valley between the two maxima, almost nonexistant for sunspots, is significantly deep for the other indices, particularly for indices originating in and above the chromosphere (corona). Occasionally, some indices show maxima several months before or after the sunspot maxima, and these can be near the photosphere, as well as in and above the chromosphere (Coronal green line index, Total Solar Irradiance, $\mathrm{Ca}$ Plage area).

\section{Years around sunspot minima}

\subsection{Plots for cycles $1-17$}

Figure 8 shows a plot of the 12-month moving averages of sunspot number $R z$ near the sunspot minima for cycles 117. As can be seen, the behaviour of $R z$ varies considerably from cycle to cycle. In cycles $2,3,5,8,9,10,11,12,14$, 15,17 , there was a single minimum and it was fairly sharp. In cycles $1,4,6,7,13,16$, the minimum was broad, extending over several months. In cycles 1 and 13, the values near minimum remained low for more than a year. One of the earliest measurements available for any other index is for the Mount Wilson Calcium Plage area, since 1915. For cycles 16 and 17, the dashed curve shows the 12-month moving averages for the Plage area. In cycle 17, the minima of $R z$ and the Plage area occur almost simultaneously, in AugustSeptember 1933. In cycle 16, the Plage area reaches a minimum in October-December 1922 and then increases thereafter, but $R z$ continues to have low values, reaching a minimum in July-September 1923. Thus, the Plage area may have an evolution different from that of sunspots. 

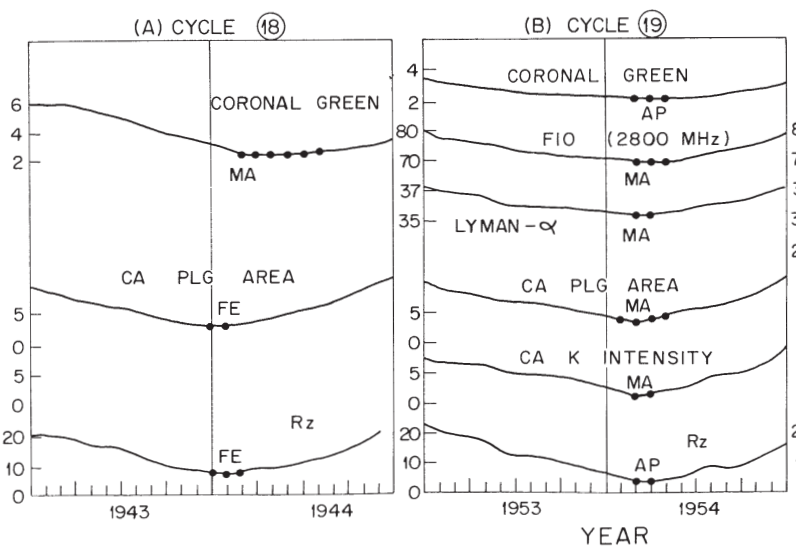

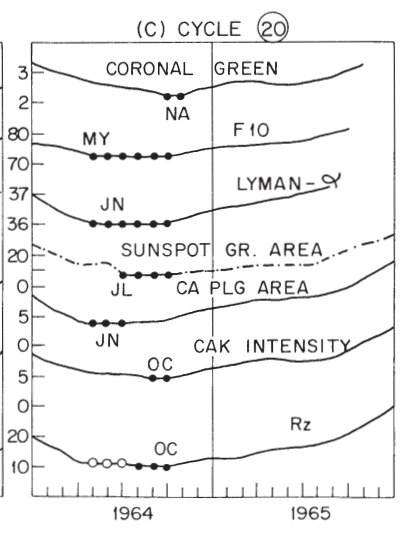

Fig. 9. Plots of the 12-month moving averages of various indices during years of sunspot minima of (A) cycle 18, 1943-1944, (B) cycle 19, 1953 1954, (C) cycle 20, 1964-1965.

\subsection{Plots for cycles $18-23$}

Figure 9 shows plots of the 12-month moving averages of various indices near the sunspot minima for (A) cycle 18, (B) cycle 19, (C) cycle 20. The indices for which data were available are:

(i) the sunspots, NGDC,

(ii) the Calcium $\mathrm{K}$ line intensity, McMath, Sacremento Peak, Big Bear Observatories, Swartz and Overbeck (1971), NGDC,

(iii) Mt. Wilson Observatory Calcium Plage Area, Foukal (1996), NGDC, and Rome Astronomical Observatory Sunspot group area, NGDC,

(iv) Lyman- $\alpha$ modelled values (Woods et al. (2000), ftp:// laspftp.colorado.edu/pub/solstice/composite_lya.dat),

(v) the $10.7 \mathrm{~cm}(2800 \mathrm{MHz})$ flux denoted as F10, NGDC, and

(vi) the Coronal green line (Fe XIV, $5303 \AA$ ) index (Rybansky et al., 1998; Ramesh, 1998), NGDC.

In cycle 18 (Fig. 9a), besides sunspot number $R z$, data for only the Ca Plage area and Coronal green line intensity index were available. The sunspot had a fairly sharp minimum around February 1944. (Values within 5\% of the minimum are also marked as minima, by full dots.) The evolution of the Plage area seems to coincide very well with that of the sunspots, in both aspects, namely the month of occurrence and the shape before and after the minimum, (similar to cycle 17 but unlike cycle 16, see Fig. 8). The Coronal green line index has a minimum at about the same month (March 1944), but the evolution after the minimum is different. Whereas $R z$ and the Plage area recover after March 1944, the Coronal line remains near the minimum for the next 6 months. This type of difference is seen later in many other cases.

In cycle 19 (Fig. 9B), the $R z$ minimum was fairly sharp, in March-April 1954. All indices, including the additional ones, $\mathrm{Ca} \mathrm{K}$ line intensity, Lyman- $\alpha$ and F10, show a minimum at about the same month, and the shapes of the evolutions are also alike. In cycle 20 (Fig. 2C), the $R z$ had a diffuse minimum during August-October 1964. The Ca K intensity index and Coronal green line index matched with sunspots. The Plage area had a minimum somewhat earlier (May 1964), but the Sunspot Group Area had minima coinciding with sunspots. The Lyman- $\alpha$ and F10 had minima earlier than $R z$. The $R z$ values for May-July 1964 were also low, though about $7 \%$ higher than the minimum. Thus, it may be concluded that $R z$, Plage area, Lyman- $\alpha$ and F10 reached almost minimum from May 1964 onwards up to October 1964. In that case, the Ca K line intensity and Coronal green line index would be considered as having a minimum with a delay of few months.

Figure 10 shows the plots for (A) cycle 21, (B) cycle 22, (C) cycle 23. The additional indices available were:

(i) Solar magnetic field represented by the Magnetic Plage Strength Index (MPSI) derived from spatially resolved magnetogram observations at the 150 -foot solar tower at Mt. Wilson Observatory (MWO) (Parker et al., 1998), full line, website http://www.astro.ucla.edu/ obs/intro. $\mathrm{html}$, and Kitt Peak line of sight magnetic field strength data (dashed line), website ftp://argo.tuc.noaa.edu/kpvt/ daily/stats/mag.dat.

(ii) Monthly counts of grouped solar flares (SGD), and the solar flare index data from the Kandilli Observatory, Istanbul, Turkey (Ataç and Ozguç, 1998, NGDC), separately for the Northern $(\mathrm{NH})$ and Southern $(\mathrm{SH}) \mathrm{Hemi}-$ spheres of the Sun.

(iii) Mg II (2800 Å, Deland and Cebula, 1993), NGDC,

(iv) Total Solar Irradiance (Frohlich and Lean, 1998), data from PMOD/WRC and VIRGO team, NGDC,

(v) He I absorption line (10830 ̊, website ftp://argo.tuc. noao.edu/kpvt/daily/stats/he.dat).

(vi) X-rays (GOES satellites), NGDC, and 
(A) CYCLE (21)
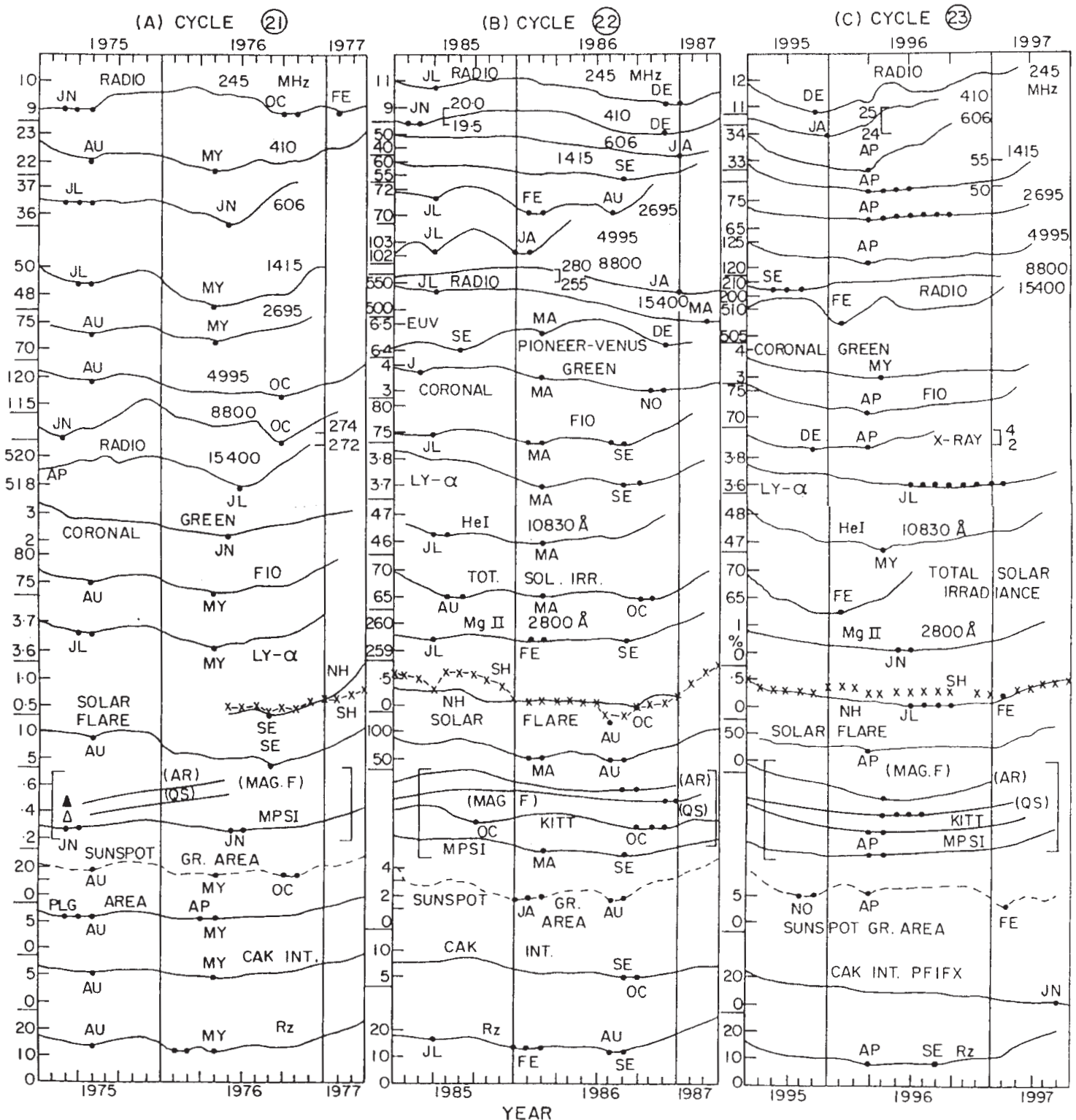

Fig. 10. Plots of the 12-month moving averages of various indices during years of sunspot minima of (A) cycle 21, 1975-1977, (B) cycle 22, 1985-1987, (C) cycle 23, 1995-1997.

(vii) Pioneer Venus Orbiter integrated EUV flux (300$1220 \AA ̊$, Brace et al., 1988; Mahajan et al., 1998, NGDC, updated data sent privately by K. K. Mahajan), and

(viii) Radio emissions at 609, 1415, 2695, 4995, 8800, $15400 \mathrm{MHz}$ (SGMR, Sagamore Hill; PALE, Palehua, Hawaii; LEAR, Learmonth, Australia; SVTO, San Vito, Italy), NGDC.

\section{Cycle 21}

In cycle 21 (Fig. 10A), the sunspot numbers had a minor minimum in August 1975 and a diffuse minimum during February-May 1976. The Calcium K intensity, Plage area and Sunspot Group Area had a similar evolution, but the Sunspot group area continued to be low until the end of 1976, though $R z$ had recouped considerably by that time. For the solar magnetic field, four different estimates were available:

(i) MPSI is the Magnetic Plage Strength Index from Mt. Wilson Observatory.

(ii) Kitt Peak gives a line of sight magnetic field strength. For the same (Kitt Peak), K. Harvey kindly sent magnetic flux values on the Sun as this flux is crossing the central meridian, separating the flux into:

(a) active regions, AR, exceeding 25 Gauss, and

(b) quiet Sun, QS, below 25 Gauss. 
In cycle 21, MPSI shows a first minimum in June-July 1975, slightly earlier than the August 1975 minimum of $R z$, and a second minimum in June-July 1976, slightly later than the May 1976 minimum of $R z$. Thus, the magnetic field lost strength earlier than $R z$ and recouped it later than $R z$. However, the active region (AR) and quiet Sun (QS) values, (12-month moving averages available only from mid-1975), showed a steady rise from 1975 onwards, quite unlike the MPSI values.

The monthly count of grouped solar flares showed a minor minimum in August 1975, tallying with that of $R z$, and a major minimum in September 1976, later than the $R z$ minimum in May 1976. Thus, solar flares disappeared later than the sunspots. The Kandilli observations of solar flare index (12-month moving averages) for the $\mathrm{NH}$ (full line) and $\mathrm{SH}$ (crosses) solar hemispheres were available only from mid1976 and both NH and SH showed a minimum in September 1976, later than the $R z$ minimum. Lyman- $\alpha$ and F10 showed minima matching with $R z$, but Coronal green line index showed only one minimum in June 1976, almost matching with $R z$. Thus, the Coronal green line seems to have a tendency of lagging behind $R z$.

A whole set of radio emission data were available in cycle 21. Almost all the emissions had a minor minimum in June-August 1975, slightly earlier than the $R z$ minimum in August 1975 (except $15400 \mathrm{MHz}$ which had a minimum in April 1975), and a major minimum in May-July 1976, almost matching with $R z$ minimum of May 1976. Exceptions were $15400 \mathrm{MHz}$, having a minor mimimum in April 1975, and $245,4995,8800 \mathrm{MHz}$, having major minima in October 1976, later than the major minimum of $R z$ in May 1976. Thus, solar radio emissions seem to lag behind $R z$.

\section{Cycle 22}

In cycle 22 (Fig. 10B), the sunspots $R z$ had two minima, one in January-March 1986 and another in August-September 1986 (besides a small inflection earlier in July 1985). The Ca K line intensity had only one minimum, in SeptemberOctober 1986, matching with sunspots. There were no Plage area data. The Sunspot Group Area had two minima matching with sunspots. In the solar magnetic field indices, MPSI had two minima, matching with sunspots. The Kitt Peak index showed two minima but far away from the $R z$ minima, the first one earlier and the second one later. Thus, Kitt Peak field disappeared earlier and revived later than $R z$. The Kitt Peak magnetic field indices AR (active region) and QS (quiet Sun) showed similar results. The solar flare indices, Mg II, Total Solar Irradiance, Lyman- $\alpha$, F10, Coronal green line and Pioneer-Venus EUV, all show good matching with sunspots, except that the second minimum is, in some cases, in November-December 1986, rather than in AugustSeptember, for $R z$. The small inflection in July 1985 for $R z$ is displayed prominently in some indices. In radio emissions, the major $R z$ minimum of January-March 1986 is missing in radio emissions $245,410,606,1415,8800,15400 \mathrm{MHz}$, and present only in 2695 and $4995 \mathrm{MHz}$. The $R z$ minimum

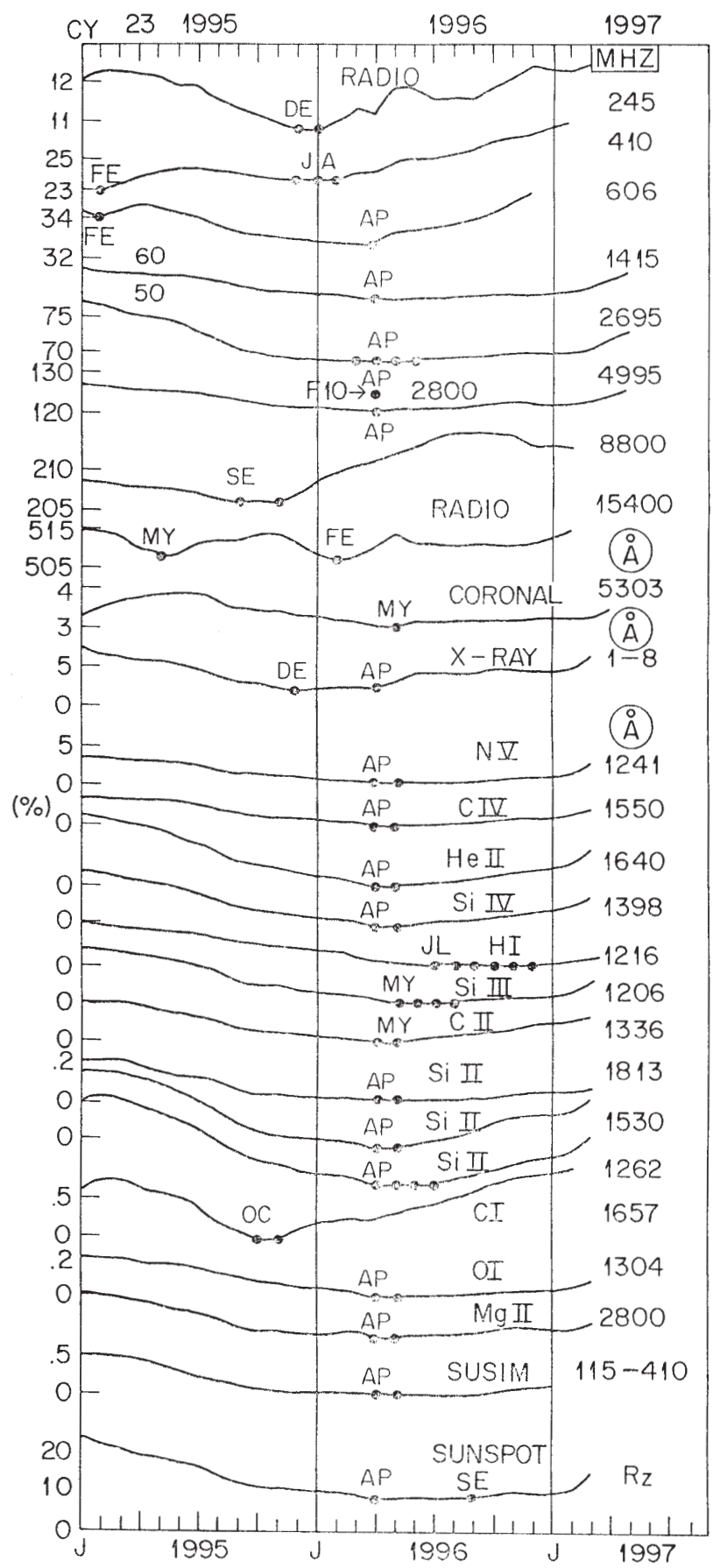

Fig. 11. Plots of the 12-month moving averages of various chromospheric line indices and coronal radio emission indices during years of sunspot minimum of cycle 23, 1995-1997.

of August-September 1986 is shifted to the end of 1986 for $245,410,606,8800$ and $15400 \mathrm{MHz}$, while the small inflection of $R z$ in July 1985 is shown prominently by these radio emissions. Thus, different radio emissions had different evolutions. 
Cycle 23

In cycle 23 (Fig. 10C), sunspots $R z$ had one prominent minimum in April 1996, but the values had a flat level until September 1996. The April 1996 minimum was reflected in many indices. Radio emissions 245, 410, 8800 and $15400 \mathrm{MHz}$ showed minima earlier, during or before January-February 1996. X-rays and Sunspot Group Area also showed similar earlier minima. Thus, these activities disappeared earlier than the $R z$. In late 1991, a whole set of data of UV spectral lines (daily values of intensities) became available from the UARS SOLSTICE observations of eleven lines, namely, Hydrogen I (Lyman- $\alpha$ ), $1216 \AA$ A Helium II, $1640 \AA$; Nitrogen V, $1240 \AA$; Oxygen I, $1304 \AA$; Carbon I, $1657 \AA$, II, $1335 \AA$, IV, $1548 \AA$; Silicon II, $1262 \AA$, II, $1530 \AA$, III, $1206 \AA$, IV, $1398 \AA$, kindly supplied to us by Dr. Thomas Woods of the University of Colorado. These lines originate in various parts of the chromosphere and the transition region and, in conjunction with the radio emissions, cover the whole solar atmosphere. In Fig. 11, the evolution of all these is shown for cycle 23. For the chromospheric spectral lines, the excitation temperatures were determined by Woods et al. (2000), taking the ratio of excitation rates for two doublets of the same super-multiplet (Zirin, 1988). In Fig. 11, the lines are arranged according to a descending order of these temperatures. Thus, N V $1241 \AA$ has a temperature of $\sim 200000 \mathrm{~K}$, while $\mathrm{Mg}$ II $2800 \AA$ has $\sim 6500 \mathrm{~K}$. The top plot of $245 \mathrm{MHz}$ has a temperature above one million degrees $\mathrm{K}$. As can be seen, almost all indices have a minimum in or near April 1996, the same as for sunspot number $R z$ (bottom plot), for which there is a broad minimum during April-September. However, there are some glaring exceptions. In the chromosphere, C I $1657 \AA$ has a minimum much earlier, in October-November 1995. The same is true for Xrays and some radio emissions. Thus, some indices weaken much earlier than the sunspots.

Regarding sunspot minima, Harvey and White (1999) made an interesting investigation for cycles 22-23. They concluded that cycle minimum is not defined solely on the basis of the occurrence of the minimum in the smoothed sunspot number, but rather by several additional parameters, including the monthly (or rotationally) averaged sunspot number, the number of regions (total, new- and old-cycle), and the number of spotless days, and recommended that the minimum between cycles 22 and 23 should be considered as to have occurred in September 1996 and not in May 1996. In Fig. 10C, we have considered the sunspot minimum as starting in April 1996, which seems to match with the minima of many other indices. (The discrepancy whether as to the sunspot minimum should be in April or May, 1996 is appearing due to the consideration of centering. We have considered 12-month moving averages as centered on the sixth month, e.g. January to December mean centered at June. Others may consider it as centered at July. The doublesmoothed means as given in McKinnon (1987) will be centered at July.) If September 1996 is considered as sunspot minimum, the minima of many indices would have occurred

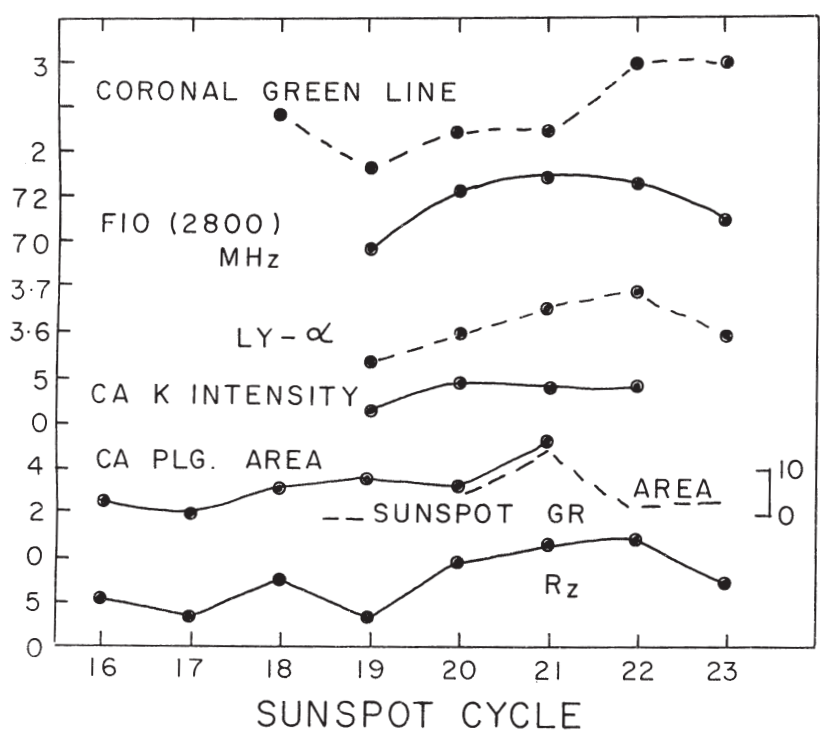

Fig. 12. Plots of the minima of some solar indices for cycles 16-23.

much earlier than sunspots. The smoothed sunspot numbers had a minimum value of 7.6 in April 1996, but the values continued to be low until September 1996 for which the value was only 8.4, almost the same as for April 1996.

5.3 Long-term variation of the minima of some solar indices

Since data for some indices are available for several sunspot cycles, it would be interesting to see if their minima have similar evolutions on a long-term time scale. Figure 12 shows a plot of the minimum values in recent cycles. The minimum of sunspot number $R z$ shows a rise from cycle 19 to cycle 22 and a fall thereafter for cycle 23. Most of the other indices show a similar pattern, but the Sunspot Group Area seems to have a decline from cycle 21 to cycles 22 and 23.

\subsection{Conclusions about years around sunspot minima}

The results about sunspot minima can be summarised as follows:

(1) The smoothed sunspot minimum can be sharp and distinct, lasting for a month or two, or can spread over several months.

(2) Among the indices originating at or near the photosphere, the $\mathrm{Ca} \mathrm{K}$ line intensity shows good matching with sunspots, but the Ca Plage area, the Sunspot Group Area, and the solar magnetic fields seem to show minima earlier than the sunspots, indicating that these activities die out first. These also show recoveries from the minima later than sunspots.

(3) Most of the other indices originating in the chromosphere and corona attain minima coincident with 
sunspot minima, but in some cases, minima earlier than sunspots are seen, while in some other cases minima occur after the sunspot minima. Thus, the energy dissipation in the upper part of the solar atmosphere sometimes lags or leads the evolution of sunspots near sunspot minimum. In a few cases, after the minimum, the indices recover faster than the sunspots.

(4) In general, the chromospheric indices seem to evolve similar to sunspots, but the evolution of coronal indices is not always similar to sunspots, and may differ considerably between themselves.

\section{General conclusions and discussion}

Based on the examination of 12-month moving averages, the general conclusion of this study is that the evolutions of various solar indices around years of sunspot maxima and minima are not always similar to that of sunspots. Lags and/or leads of several months are often seen. The observed differences between the chromospheric and coronal indices have important implications for solar dynamics. Harvey (1992) reported that the solar magnetic field relationship with photospheric total irradiance was different from that with chromospheric and coronal radiations, though the correlation between magnetic field and F10 was very good. Ivanov et al. (1998) used cross-correlation for short-term (27-day) variations and showed a similar result, namely that the solar global magnetic field had a relationship with photospheric radiation which was different from that with (upper) chromospheric and coronal radiations. Ataç and Ozguç (1998) showed similar dissimilarities between various indices (no good agreement with Total Solar Irradiance, etc.). Particularly interesting were the differences in the behaviour of indices of the $\mathrm{NH}$ and $\mathrm{SH}$ solar hemispheres, where the solar flare activity drifted between the two hemispheres every two years from 1988 to 1993 . The N-S asymmetry is manifested in several features of solar activity (detailed references in Ataç and Ozguç, 1998). For example, Joshi (1995) had shown the existence of a constant and persistent N-S asymmetry of sunspot groups, $\mathrm{H}$ Alpha flares and active prominences during the maximum of cycle 22 (1989-1991) (see also Garcia, 1990). Thus, the convection of the solar energy from the solar interior to the photosphere, chromosphere and corona is not similar and is much more complicated than the traditional blocking effect (Ivanov et. al., 1998).

Since all solar indices attain a maxima near sunspot maximum and minima near sunspot minimum, the long-term correlation between all indices is very high. On a shortterm scale, different indices have different time scales (seconds, minutes for flares, 1-27 days for sunspot evolution and movements, etc.) and different locations on the photosphere and hence, considerable differences in the evolution patterns are expected and seen, even when whole-disc measurements are considered. For intermediate time scales (moving averages over several months), only the background levels should be relevant and one would expect that these would be similar for all indices. But it seems that some of the short-term effects spill over in the intermediate-term time scale. Differences in hemispheric evolutions probably play a major role, even though full-disc values are considered. Donnelly et al. (1986) and Ataç and Ozguç (1998) have illustrated the spill-overs for averages over a few months. Dissimilarities even in 12-month moving averages are surprising.

During sunspot minima, the dissimilarity of the behaviour of the Ca Plage (or sunspot) areas on one side and the CA K line intensity and sunspots on the other indicates that the areas have a partially independent evolution pattern (intense spots not necessarily bigger in size). Also, the magnetic fields die out earlier and recover later, indicating that the conditions to produce and sustain the magnetic structures are more demanding than those needed for producing sunspots. Once the energy from the interior of the Sun emerges and reaches higher altitudes, its evolution and dissipation there seems to be affected considerably by solar atmospheric dynamics, and similarity with surface activity is diminished. The intriguing aspect is that the dissimilarity is not similar for all radio emissions, nor is there any clear-cut dependence on solar altitudes, though there is a slight indication that the upper corona may show larger dissimilarities.

Acknowledgements. Thanks are due to H. Coffey and E. Erwin for guiding through the NOAA website and indicating websites for other data (including the NSO/Kitt Peak data used here which are produced cooperatively by NSF/NOAO, NASA/GSFC, and NOAA/SEL)., to T. Woods at the University of Colorado (Boulder, Colorado, USA) for providing the solar UV irradiances from the UARS SOLSTICE observations and for useful discussions and suggestions, to K. K. Mahajan for supplying updated Pioneer Venus Eipe data privately, to R. K. Ulrich (MPSI data) and D. McMullin (CELIAS/SEM, SOHO data) for giving access to their websites, and to the other innumerable workers who are responsible for producing the scientific data used in this analysis. This work was partially supported by FNDCT, Brazil under contract FINEP-537/CT.

Topical Editor E. Antonacci thanks I. Jocelyn and G. Godoli for their help in evaluating this paper.

\section{References}

Ataç, T. and Ozguç, A.: Flare index of solar cycle 22, Solar Phys. 180, 397-407, 1998.

Brace, L. H., Hoegy, W. R., and Theis, R. F.: Solar EUV measurements at Venus based on photoelectron emission from the Pioneer Venus Langmuir probe, J. Geophys. Res. 93, 7282-7296, 1988.

Chapman, G. A., Cookson, A. M., and Dobias, J. J.: Observations of changes in the bolometric contrast of sunspots, Astrophys. J., 432, 403-408, 1994.

Chapman, G. A., Cookson, A. M., and Dobias, J. J.: Variations in total solar irradiance during solar cycle 22, J. Geophys. Res. 101, 13 541-13 548, 1996.

DeLand, M. T. and Cebula, R. P.: Composite Mg II solar activity index for solar cycles 21 and 22, J. Geophys. Res., 98, 12 809$12823,1993$. 
Donnelly, R. F., Harvey, J. W., Sheath, D. F., and Repoff, T. P.: Temporal characteristics of the solar UV flux and HE I line at 1083 nm, J. Geophys. Res., 90, 6267-6273, 1985.

Donnelly, R. F., Hinteregger, H. E., and Sheath, D. F.: Temporal variations of solar EUV, UV, and $10830 \AA$ radiations, J. Geophys. Res., 91, 5567-5578, 1986.

Foukal, P.: The Behavior of Solar Magnetic Plages Measured from Mt. Wilson Observations between 1915-1984, Geophys. Res. Lett., 23, 2169-2172, 1996.

Frohlich, C. and Lean, J.: The Sun's total irradiance: Cycles, trends and related climate change uncertainties since 1976, Geophys. Res. Lett., 25, 4377-4380, 1998.

Garcia, H. A.: Evidence of solar-cycle evolution of North-South flare asymmetry during cycles 21 and 22, Solar Phys., 127, 185197, 1990.

Harvey, K. L.: Measurements of Solar Magnetic Fields as an Indicator of Solar Activity Evolution, in: Proceedings of SOLERS22: Proceedings of the Workshop on the Solar Electromagnetic Radiation Study for Solar Cycle 22, (Ed) Donnelly, R. F., U. S. Department of Commerce, SEL NOAA ERL, 113-129, 1992.

Harvey, K. L. and White, O. R.: What is solar cycle minimum?, J. Geophys. Res., 104, 19759-19764, 1999.

Heath, D. F. and Schlesinger, B. M.: Temporal variability of UV solar irradiance from $160-400 \mathrm{~nm}$ over periods of the evolution and rotation regions from maximum to minimum phases of the sunspot cycle, in: IRS 84: Current problems in Atmospheric Radiation, Proceedings of the International Radiation Symposium, Perugia, Italy, (Ed) Fiocco, G., 315-319, A. Deepak, Hampton, Va., 1984.

Hinteregger, H. E., Fukui, K., and Gilson, B. G.: Observational, reference, and model data on solar EUV, from measurements on AE-E, Geophys. Res. Lett., 8, 1147-1150, 1981.

Ivanov, E. V., Obridko, V. N., and Ananyev, I. V.: Variations of solar irradiance, $10.7 \mathrm{~cm}$ radio flux, He I $10830 \AA$ equivalent width, and global magnetic field intensity and their relation to largescale solar magnetic field structure, Solar Phys., 177, 217-228, 1998.

Joshi, A.: Asymmetries during the maximum phase of solar cycle 22, Solar Phys., 157, 315-324, 1995.

Kane R. P., Hari Om Vats, and Sawant, H. S.: Short-term periodicities in the time series of solar radio emissions at different solar altitudes, Solar Phys., 201, 181-190, 2001.

Lean, J.: Solar ultraviolet irradiance variations: A review, J. Geophys. Res., 92, 839-868, 1987.

Mahajan, K. K., Hari Om Upadhyay, Sethi, N. K., Hoegy, W. R.,
Pesnell, W. D., and Brace, L. H.: Pioneer Venus Orbiter measurements of solar EUV flux during solar cycles 21 and 22, Solar Phys., 177, 203-216, 1998.

McKinnon, J. A.: Sunspot numbers 1610-1985, UAG Report 95, pp 112, NOAA Boulder, Colorado, USA, 1987.

Mount, G. and Rottman, G.: The solar spectral irradiance 1200$3184 \AA$ A near solar maximum: 15 July 1980, J. Geophys. Res., 86, 9193-9198, 1981.

Mount, G. H., Rottman, G. J., and Timothy, J. G.: The solar spectral irradiance 1200-2550 ̊ at solar maximum, J. Geophys. Res., 85, 4271-4274, 1980.

Parker D. G., Ulrich, R. K., and Pap, J. M.: Modeling solar UV variations using Mount Wilson Observatory indices, Solar Phys., 177, 229-241, 1998.

Ramesh, K. B.: A correlative study of green coronal intensity with other solar indices, Solar Phys., 177, 311-319, 1998.

Rottman, G. J.: Rocket measurements of the solar spectral irradiance during solar minimum, J. Geophys. Res., 86, 6697-6705, 1981.

Rottman, G. J.: Solar cycle variation of ultraviolet, 120-200 nm irradiance measured by the solar mesosphere explorer, 1982 through 1985, Eos Trans. AGU, 66, 1009, 1985.

Rottman, G. J. and London, J.: Solar UV irradiance observations of 27-day variations and evidence for solar cycle variations, in: IRS 84: Current problems in Atmospheric Radiation, Proceedings of the International Radiation Symposium, Perugia, Italy, (Ed) Fiocco, G., pp. 320-324, A. Deepak, Hampton, Va., 1984.

Rybansky, M., Rusin, V., and Minarovjech, M.: The green coronal index and soft X-ray flux, Solar Phys., 177, 305-310, 1998.

Schwabe, S. H.: Die Sonne, Astronomische Nachrichten 20, 473, 283-288, 1843.

Schwabe, S. H.: Sonnen-Beobachtung im Jahr 1843, Astronomische Nachrichten 21, 495, 234-235, 1844.

Swartz, W. E. and Overbeck, R.: The Solar Ca II Plage Index, Pennsylvania State University Ionosphere Research Laboratory Report 373(E), 8 October, 1971.

Waldmeier, M.: The sunspot activity in the years 1610-1960, Schulthess \& Company AG, Zürich, 1961.

Woods, T. N., Tobiska, W. K., Rottman, G. J., and Worden, J. R.: Improved solar Lyman alpha irradiance modeling from 1947 through 1999 based on UARS observations, J. Geophys. Res., 105, 27 195-27 215, 2000.

Zirin, H.: Chapter 7. The Chromosphere, Astrophysics of the Sun, Cambridge University Press, 1988. 